\title{
Effects of efavirenz and tenofovir on bone tissue in Wistar rats
}

\author{
Agnieszka Matuszewska ${ }^{1, A-F}$, Beata Nowak ${ }^{1, A-F}$, Anna Nikodem 2,B,C,E,F, Diana Jędrzejuk 3,, ${ }^{3, F}$, \\ Danuta Szkudlarek ${ }^{4,, F}$, , Krzysztof Zduniak ${ }^{4, C, F}$, Jarosław Filipiak ${ }^{2, C, E, F}$, Marta Sznadruk-Bender ${ }^{1, C, E, F}$, \\ Tomasz Tomkalski ${ }^{5, C, E, F}$, Ireneusz Ceremuga ${ }^{6, B, C, E, F}$, Marek Bolanowski ${ }^{3, E, F}$, Adam Szeląg ${ }^{1, E, F}$ \\ ${ }^{1}$ Department of Pharmacology, Wroclaw Medical University, Poland \\ ${ }^{2}$ Division of Biomedical Engineering and Experimental Mechanics, Wroclaw University of Technology, Poland \\ ${ }^{3}$ Department of Endocrinology, Diabetes and Isotope Therapy, Wroclaw Medical University, Poland \\ ${ }^{4}$ Department of Pathology, Wroclaw Medical University, Poland \\ ${ }^{5}$ Department of Endocrinology, T. Marciniak Lower Silesian Specialist Hospital, Poland \\ ${ }^{6}$ Department of Medical Biochemistry, Wroclaw Medical University, Poland \\ A - research concept and design; $\mathrm{B}$ - collection and/or assembly of data; $\mathrm{C}$ - data analysis and interpretation; \\ $\mathrm{D}$ - writing the article; $\mathrm{E}$ - critical revision of the article; $\mathrm{F}$ - final approval of the article
}

\section{Address for correspondence \\ Beata Nowak}

E-mail: beata.nowak@umed.wroc.pl

\section{Funding sources}

This study was supported by Wroclaw Medical University (grant No. ST A080.17.035).

Conflict of interest

None declared

Received on March 27, 2020

Reviewed on June 14, 2020

Accepted on September 20, 2020

Cite as

Matuszewska A, Nowak B, Nikodem A, et al.

Effects of efavirenz and tenofovir on bone tissue in Wistar rats. Adv Clin Exp Med. 2020;29(11):1265-1275. doi:10.17219/acem/127684

D0I

10.17219/acem/127684

Copyright

Copyright by Author(s)

This is an article distributed under the terms of the

Creative Commons Attribution 3.0 Unported (CC BY 3.0)

(https://creativecommons.org/licenses/by/3.0/)

\section{Abstract}

Background. Clinical trials indicate an increased risk of osteoporosis and bone fractures in people infected with human immunodeficiency virus (HIV). The pathogenesis of bone disturbances in HIV-positive patients is unknown, but it is suggested that antiretroviral drugs may be involved.

Objectives. To assess the effects of efavirenz (EF) and tenofovir (T) on bone remodeling in rats.

Material and methods. The study involved 36 male Wistar rats divided into 3 groups, receiving normal saline (control group - group C), efavirenz (group EF) or tenofovir disoproxil (group T).

Results. After 24 weeks of the study, the following observations were made: In blood serum of the EF group compared to group C, there were increased levels of tartrate-resistant acid phosphatase form $5 b$ (TRAP) and inorganic phosphorus. In the densitometric examination, group T showed a lower total body (TB) bone mineral density (BMD) than group C. In the immunohistochemical assessment, group EF showed a higher intensity and extension of anti-tartrate resistant acid phosphatase antibodies (abTRAP) compared to group C. In the histopathological examination of the second lumbar vertebra (L2), group EF showed a lower bone surface/ volume ratio (BS/BV) and higher trabecular thickness (Tb.Th) than the control group. In the histopathological examination of the femur, a lower bone surface/tissue volume (BS/TV) and lower trabecular number (Tb.N) were found in group T compared to in group C. A lower value of the Young's modulus was observed in the four-point bending trial in groups $E F$ and $T$ compared to group $C$.

Conclusions. The results of this study indicate that EF affects bone microarchitecture and leads to impaired biomechanical properties of bones in rats. Additionally, the negative effect of T on bone tissue was confirmed.

Key words: bone, rat model, efavirenz, tenofovir, antiretroviral drug 


\section{Introduction}

Human immunodeficiency virus (HIV) and acquired immunodeficiency syndrome (AIDS) constitute an increasing epidemiological problem. It is estimated that about 36.9 million people in the world are HIV-positive, including 1.8 million children below 15 years of age. ${ }^{1}$ Highly active antiretroviral therapy (HAART) is the only method of treatment of HIV infections. It is currently administered to neonates, infants and young children infected with HIV.

Effective antiretroviral treatment reduces HIV RNA viremia and the incidence of opportunistic infections and tumors in HIV-positive patients, thus extending their survival and reducing the mortality rate. The necessity of chronic antiretroviral therapy is associated with increasing challenges in the form of chronic drug-related adverse effects, including osteoporosis. A detailed knowledge of possible adverse effects of a drug allows medical teams to optimize the benefits of antiretroviral therapy while minimizing the risks associated with long-term treatment. The necessity of treating children requires assessments of the effects of the drugs administered on the processes of growth, development and maturation of individual organs.

Reduced bone mineral density (BMD), decreases in bone mass and changes in bone microarchitecture are reported in HIV-positive children and adolescents. ${ }^{2,3}$ Lower BMD in HIV-positive children is associated with decreases in vitamin $\mathrm{D}$ levels and elevated parathyroid hormones (PTH). ${ }^{4,5}$ T-cell activation with HIV infection decreases the number of osteogenic precursors, leading to lower peak bone mass and bone strength. ${ }^{6}$ As a consequence, HIV-infected children do not reach the same peak bone mass as found in HIV-negative children, and experience an increased frequency of fractures. ${ }^{7}$ In animal models of HIV-1, transgenic rats bone loss is a consequence of enhanced osteoclastic bone resorption expressed by an elevated RANKL/OPG ratio. ${ }^{8}$ In addition, Ofotokun et al. reported that HIV directly infects circulating osteoclast precursors, enhancing their differentiation and migration to bones, which leads to enhanced bone resorption. ${ }^{9}$ On the one hand, bone loss is a direct consequence of HIV infection and AIDS-associated diseases such as muscle wasting, kidney disease and hypogonadism. An effective antiretroviral treatment, causing viral suppression, should reduce the bone mass loss associated with chronic inflammation. ${ }^{10}$ On the other hand, studies of adult patients demonstrate that some antiretroviral treatment schemes result in intensified bone mass loss, rather than reduced. ${ }^{11-15}$ Low BMD is diagnosed in HAART-treated people 2.5 times more often than those receiving no HAART. Moreover, $\mathrm{BMD}$ is reduced by as much as $2-6 \%$ during the initial 2 years of antiretroviral therapy. ${ }^{16}$ Overall, the fracture rate with HIV infection is 2-6 times higher than in the general population. ${ }^{9}$ Shiau et al. reported that BMD decreases persist in HIV-infected children even in the immunologically stable phase of HIV infection. ${ }^{2}$ Various antiretroviral drugs seem to have different effects on bone metabolism.

The results of previous studies on the effects of antiretroviral medication, including efavirenz (EF) and tenofovir $(\mathrm{T})$, on bones in children are inconclusive. Some authors have reported reduced BMD in children treated with EF and T. ${ }^{17}$ On the other hand, it has been reported that changing the HAART scheme from lopinavir/ritonavir to EF is associated with higher whole-body BMD. ${ }^{18}$ However, Dave et al. ${ }^{19}$ reported an unfavorable effect of EF on bones, demonstrating that exposure to EF as a part of the HAART scheme in young HIV-positive patients (aged 30-40 years) is independently correlated with lower total hip BMD compared to HAART-naive participants.

Gafni et al. reported reduced BMD in children treated with tenofovir as a part of the HAART scheme. ${ }^{20}$ However, other authors have observed no BMD reduction in children treated with the tenofovir-containing HAART scheme. . $^{3,21,22}$

Bone mass acquired in childhood and adolescence in a main factor influencing peak adult bone mass, which is a crucial determinant of future osteoporosis and fracture. A $10 \%$ increase in peak bone mass delays the onset of osteoporosis by 13 years. ${ }^{23}$ Therefore it is extremely important to identify strategies that will allow bone mass deterioration to be minimized in children with HIV.

Discrepancies in the results of the aforementioned studies may stem from non-homogeneous study groups, various stages of HIV infection and the use of polytherapy (which precludes monotherapy studies). For that reason, studies on an animal model are reasonable, as they may allow assessment of the effect of individual drugs on bone metabolism. A rat skeleton grows throughout the lifetime of the animal. ${ }^{8}$

The purpose of this study was to assess the effects of monotherapy with EF (non-nucleoside reverse transcriptase inhibitor - NNRTI) and T (nucleozide reverse transcriptase inhibitor - NRTI) on bone remodeling in rats. As the significance of bone loss associated with HAART in HIV-patients for fracture risk is unclear, ${ }^{24}$ we planned to examine bone mechanical properties with a four-pointbending test.

\section{Material and methods}

\section{Ethics, the animal model and the experimental design}

The study protocol was approved by the Local Ethics Commission for animal experiments (approval No. LKE 41/2017). All the procedures involving animals performed during the study were in accordance with the ethical standards and practices of the institution where the study was conducted.

The study was conducted on 36 male albino Wistar rats, aged 8 weeks, weighing 240-290 g (Animal Research 
Center, Wroclaw Medical University, Poland). Throughout the experiment, the rats were kept in pairs in cages with free access to water and standard feed, a diurnal 12:12 light-to-dark cycle and at a constant ambient temperature $\left(21-23^{\circ} \mathrm{C}\right)$. The feed (Labofeed; Morawski Feed Company, Kcynia, Poland) contained $0.95 \%$ calcium, $0.75 \%$ phosphorus and $800 \mathrm{IU} / \mathrm{kg}$ of vitamin D.

After a one-week adaptation period, the animals were randomly divided into 3 groups (12 animals in each group): a control group (group C), receiving saline solution $(4 \mathrm{~mL} / \mathrm{kg})(0.9 \%$ sodium chloride from B. Braun Melsungen AG, Melsungen, Germany), group EF, receiving $25 \mathrm{mg} / \mathrm{kg}$ of efavirenz ${ }^{25}$ (Stocrin; Merck Sharp Dohme Ltd., Hoddesdon, UK) and group T, receiving $15 \mathrm{mg} / \mathrm{kg}$ of tenofovir disoproxil ${ }^{26}$ (Teva Operations Poland sp. z o.o., Kraków, Poland). The saline solution and study drugs were administered daily for 24 weeks using gastric tubes.

Body weights were checked once daily throughout the 24-week experimental period. In weeks 12 and 24, blood samples for serum isolation were collected. The serum was separated with centrifugation (at $1500 \times \mathrm{g}$ ) and then stored at $-70^{\circ} \mathrm{C}$ until required for laboratory tests. In weeks 12 and 24 the rats were anesthetized intraperitoneally (i.p.) with ketamine $(50 \mathrm{mg} / \mathrm{kg})$ and diazepam (3 mg/kg). Their BMD was measured with dual-energy Xray absorptiometry (DXA).

In week 24, the animals were euthanized under general anesthesia with ketamine (60 mg/kg i.p.) and xylazine (10 mg/kg i.p.). Tibial and femoral bones and second lumbar vertebrae (L2) were removed for further tests. The right tibial and femoral bones were stored at $-70^{\circ} \mathrm{C}$; the left tibial and femoral bones, as well as the L2 were fixed in buffered formaldehyde for further histological examination.

\section{Macrometric bone measurements}

The carefully cleaned right femurs and tibias were weighed on electronic scales (Radwag AS 60/220/C/; Radwag, Radom, Poland). The length and diameter of both bones were measured using electronic calipers (Pro sp. z o.o., Bielsko-Biała, Poland) with $0.01 \mathrm{~mm}$ resolution.

The femoral index was calculated from the formula (eq. 1):

$$
\text { femoral index }=\frac{\text { femur mass }[\mathrm{g}]}{\text { body weight }[\mathrm{g}]} \times 100 \%
$$

The tibial index was calculated from the formula (eq. 2):

$$
\text { tibial index }=\frac{\text { tibia mass }[\mathrm{g}]}{\text { body weight }[\mathrm{g}]} \times 100 \%
$$

\section{Dual-energy X-ray absorptiometry}

The BMD of the total body (TB), femurs and tibias was measured in vivo by trained examiners using DXA (Hologic Discovery DXA System; Hologic Inc., Marlborough,
USA) with software for small animals. The BMD of the lumbar vertebrae (L1-L4) was measured ex vivo after the spine was isolated along with the ligaments and spinal muscles. The results were obtained as grams of mineral content per square centimeter of bone area $\left[\mathrm{g} / \mathrm{cm}^{2}\right]$. The scanner was calibrated daily using a phantom provided by the manufacturer.

\section{Bone histological and immunohistochemical examination}

Rat second lumbar vertebrae, left femurs and left tibias were cleaned, and then bones were fixed in $10 \%$ neutral buffered formalin and decalcified in $10 \%$ neutral buffered EDTA solution. The EDTA solution was changed once after $24 \mathrm{~h}$. Second lumbar vertebrae, the metaphyseal and epiphyseal of the distal femur, and proximal tibia were harvested, embedded in paraffin and cut into $5-\mu$ m-thick slides. The slides were stained using the standard hematoxylin and eosin (H\&E) method and scanned using the Hamamatsu NanoZoomer v. 2.0 histological slide scanner with NDP.scan SQ v. 1.0 software (Hamamatsu Photonics K.K., Iwata, Japan). A scanned area of at least $1.5 \mathrm{~mm}^{2}$ (range: $1.5-4 \mathrm{~mm}^{2}$ ) from each sample was exported to a TIFF file. The TIFF files were analyzed with ImageJ v. 1.52 software (National Institutes of Health, Bethesda, USA). The histopathological examination was consistent with the standardized nomenclature, symbols and units for bone histomorphometry as updated in 2012. ${ }^{27}$

The immunohistochemical examination was completed using paraffin-embedded $4-\mu$ m-thick sections of the L2 and anti-tartrate resistant acid phosphatase antibody (abTRAP) and recombinant anti-alkaline phosphatase antibody (abAP) (both from Abcam, Cambridge, UK). The specimens were stained according to the instructions provided by the manufacturer, using Autostainer Link 48 equipment (Dako GmbH, Glostrup, Denmark). abTRAP was used in a dilution of $0.5 \mu \mathrm{g} / \mathrm{mL}$ and abAP in a dilution of 1:500. Bound antibodies were assayed using the Dako EnVision $^{\text {TM }}$ FLEX detection system. Additional abTRAP and abAP staining was assessed quantitatively using 1-3 point scoring:

Intensity: score 1 - poor; score 2 - medium; score 3 - strong;

Extension: score 1 - from 0.5 to $10 \%$; score 2 - from 11 to $50 \%$; score 3 - over $51 \%$.

According to the recommendation from the International Ad Hoc Expert Panel, ${ }^{28}$ a negative reagent control (NRC) was used, and the primary antibody was replaced with unspecific immunoglobulins at the same concentration.

\section{Laboratory determinations}

The serum obtained through centrifuging the blood was stored at $-80^{\circ} \mathrm{C}$ until the tests. The levels of beta C-terminated telopeptide of type I collagen (CTX), 
osteoclast-derived tartrate-resistant acid phosphatase form $5 \mathrm{~b}$ (TRAP) and osteocalcin were determined with commercially available enzyme-linked immunosorbent assay (ELISA) kits, following the manufacturer's instructions (all from Immunodiagnostic Systems Limited, Boldon, UK). The level of insulin-like growth factor 1 (IGF-1) was likewise determined using a commercially available kit in accordance with the manufacturer's instructions (CloudClone Corp., Houston, USA).

A certified laboratory, using Architect plus ci4100 equipment (Abbott Laboratories, Chicago, USA) with commercial tests (also from Abbott), determined the levels of total calcium, inorganic phosphorus and creatinine in the serum samples.

\section{Biomechanical testing}

The right-side femurs were cleaned off, then the epiphysis of each femur was placed in aluminum alloy tubes with a diameter of $11 \mathrm{~mm}$, and then fixed with self-polymerizing glue Duracryl ${ }^{\circledR}$ Plus (Spofa Dental Inc., Jičín, Czechy) (Fig. 1). We measured the mechanical properties of the right-side femurs by performing a four-point bending Samples were loaded at the rate of $1 \mathrm{~mm} / \mathrm{min}$. The loading points and the dimensions are shown in Fig. 2. For the test reported in this paper, $\mathrm{a}=24 \mathrm{~mm}$ test on 858 MTS MiniBionix ${ }^{\circledR}$ equipment (MTS Systems Corporation, Eden Prairie, USA) (Fig. 2) and $b=20 \mathrm{~mm}$. Based on the strengthdeflection arrow characteristics, the following parameters were determined for each sample: the longitudinal elasticity modulus (Young's modulus, E), flexural strength (Rz) and rigidity $(\mathrm{k})$.

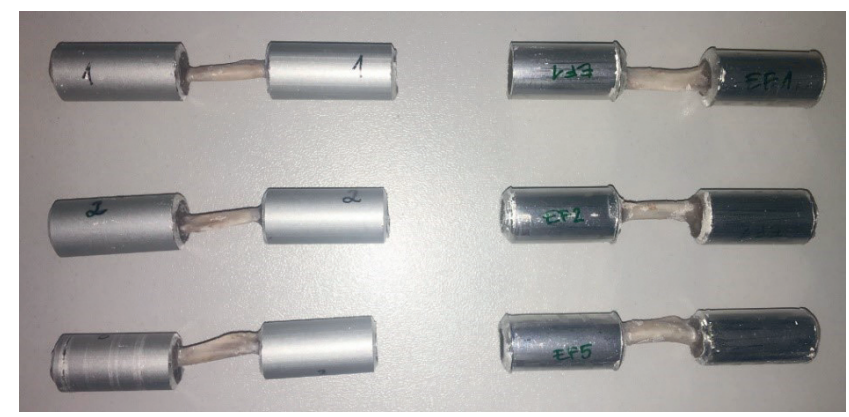

Fig. 1. Samples prepared for the four-point bending test

Young's modulus (E) was calculated from Formula 1:

$$
E=\frac{P \times b^{2}}{6 \times f^{1} \times b}(3 a+2 b)
$$

where E is Young's modulus [GPa]; $\mathrm{a}$ and $\mathrm{b}$ are the distances between supports $[\mathrm{m}]$; $\mathrm{P}$ is maximum strength $[\mathrm{N}]$; $\mathrm{f}_{1}$ is the deflection arrow $[\mathrm{m}]$ and is the moment of inertia $\left[\mathrm{m}^{4}\right]$. Flexural strength $(\mathrm{Rz})$ was determined according to Formula 2.

$$
\mathrm{Rz}=\frac{\mathrm{Mg}}{\mathrm{W}_{\mathrm{z}}}
$$

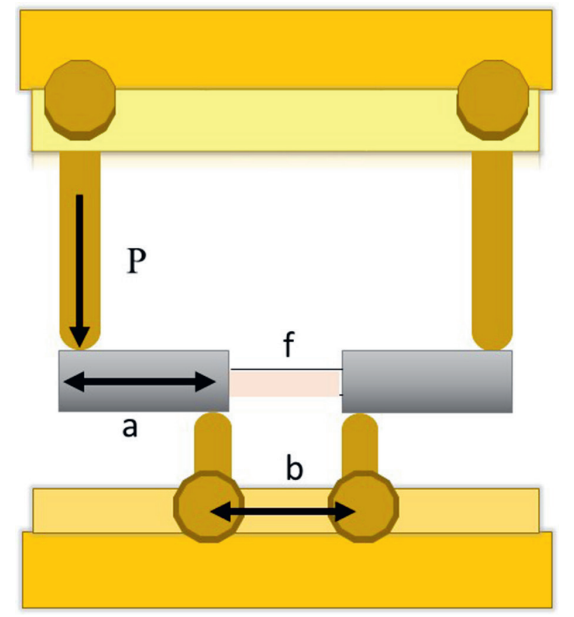

Fig. 2. The measuring system for the four-point bending test of femurs

where flexural strength $[\mathrm{MPa}] ; \mathrm{M}_{\mathrm{g}}$ is the bending moment $[\mathrm{Nm}]$; and $\mathrm{W}_{\mathrm{Z}}$ is the flexural strength index $\left[\mathrm{m}^{3}\right]$.

The rigidity $(\mathrm{k})$ of a sample was calculated from Formula 3:

$$
\mathrm{K}=\mathrm{E} \times \mathrm{I}
$$

where $\mathrm{k}$ is the rigidity to bending $\left[\mathrm{Nm}^{2}\right]$; $\mathrm{E}$ is Young's modulus [GPa]; and I is the moment of inertia of the sample cross-section $\left[\mathrm{m}^{4}\right]$.

\section{Statistical analysis}

The parameters studied were expressed as means \pm standard deviation (SD). The statistical analysis was completed using STATISTICA software v. 12 (StatSoft, Inc., Tulsa, USA). A one-way analysis of variance (ANOVA) with a post hoc least significant difference (LSD) test was used to determine significant differences between the 3 study groups. The level of significance was set at $\mathrm{p}<0.05$.

\section{Results}

\section{Body weight}

The results are presented in Table 1 . No differences in body weight were observed on day 1 between group $C$ and the groups EF and T. The groups were compared in terms of body weight throughout the entire experiment, and on the last day of the experiment we found no significant differences among the groups.

\section{Macrometric measurements of bones}

The results are presented in Table 1 . The femoral indices were lower in groups EF and T than in group $C(p=0.006$ and $\mathrm{p}=0.001$, respectively); the weights of the femurs were likewise lower in groups $\mathrm{EF}$ and $\mathrm{T}$ than in group $\mathrm{C}$ (both $\mathrm{p}=0.001$ ). The tibial indices, the weight and length 
of the tibias were lower in group EF compare to group $\mathrm{C}$ ( $\mathrm{p}=0.04, \mathrm{p}=0.003, \mathrm{p}=0.03$, respectively). Mid-femoral diameters were lower in groups EF and $\mathrm{T}$ than in group $\mathrm{C}$ ( $p=0.025$ and, $p=0.004$, respectively). The mid-tibial diameter was lower in group $\mathrm{T}$ compared to group $\mathrm{C}(\mathrm{p}=0.003)$.

\section{Dual-energy X-ray absorptiometry (DXA)}

The results are presented in Table 2. After 12 weeks, no differences were found between the study groups in terms of $\mathrm{BM}$ of the TB, femurs or tibias. After 24 weeks, we detected a lower TB BMD in group $\mathrm{T}$ compared to group $\mathrm{C}$ $(p=0.0043)$. The BMD of the lumbar spine L1-L4 was not different between the study groups at the end of the experiment.

\section{Histopathological and immuno- histochemical examinations}

The results are presented in Table 3 and Fig. 3 and 4 . In the histopathological assessment of the L2, the bone surface/volume ratio $(\mathrm{BS} / \mathrm{BV})$ was lower in group EF compared to group $C(p=0.014)$. A higher trabecular thickness (Tb.Th) was also observed in group EF compared to group $C(p=0.025)$. The immunohistochemical examination of the L2 demonstrated a higher intensity and extension of abTRAP in group EF than in group $C$ ( $\mathrm{p}=0.04, \mathrm{p}=0.02$, respectively). In group $\mathrm{T}$, increased extension of recombinant abAP compared to group $\mathrm{C}$ was at the border of statistical significance $(\mathrm{p}=0.054)$.

The histopathological examination of the femurs demonstrated a lower bone surface/tissue volume (BS/TV) and a lower number of trabeculae (Tb.N) in group $\mathrm{T}$ compared with group $\mathrm{C}(\mathrm{p}=0.014, \mathrm{p}=0.04$, respectively). Moreover, trabecular separation (Tb.Sp) was higher in group $\mathrm{T}$ compared to $\mathrm{C}(\mathrm{p}=0.009)$.

\section{Serum parameters}

The results are presented in Table 4 . After 12 weeks, no statistically significant differences were found in CTX and osteocalcin levels between group $\mathrm{C}$ and groups EF and T. After 24 weeks, higher serum levels of TRAP, calcium and inorganic phosphorus, and a lower osteocalcin level were observed in group EF compared to $\mathrm{C}(\mathrm{p}=0.002$, $\mathrm{p}=0.013, \mathrm{p}=0.0003$ and $\mathrm{p}=0.008$, respectively).

Table 1. The effect of long-term administration of efavirenz and tenofovir on body weight and macrometric parameters of bones (one-way ANOVA with post hoc LSD test)

\begin{tabular}{|c|c|c|c|c|}
\hline \multicolumn{2}{|c|}{ Parameter } & Group C & Group EF & Group T \\
\hline \multirow{2}{*}{ Body weight [g] } & week 1 & $270.0 \pm 13.5$ & $266.2 \pm 11.0$ & $257.8 \pm 12.8$ \\
\hline & week 24 & $468.7 \pm 30.5$ & $440.7 \pm 27.3$ & $450.2 \pm 33.9$ \\
\hline \multirow{4}{*}{ Femur } & femoral index & $0.3353 \pm 0.0328$ & $0.3068 \pm 0.0161^{*}$ & $0.3013 \pm 0.0171^{*}$ \\
\hline & femur weight $[\mathrm{g}]$ & $1.558 \pm 0.157$ & $1.349 \pm 0.060^{*}$ & $1.358 \pm 0.141^{*}$ \\
\hline & femur length [mm] & $38.061 \pm 1.626$ & $39.093 \pm 1.676$ & $38.428 \pm 1.584$ \\
\hline & mid-femoral diameter [mm] & $3.963 \pm 0.171$ & $3.762 \pm 0.151^{*}$ & $3.702 \pm 0.256^{*}$ \\
\hline \multirow{4}{*}{ Tibia } & tibial index & $0.2367 \pm 0.0204$ & $0.2201 \pm 0.0129^{*}$ & $0.2271 \pm 0.02023$ \\
\hline & tibial weight $[\mathrm{g}]$ & $1.108 \pm 0.106$ & $0.968 \pm 0.047^{*}$ & $1.023 \pm 0.121$ \\
\hline & tibia length [mm] & $43.611 \pm 0.856$ & $42.583 \pm 1.090^{*}$ & $43.603 \pm 1.186$ \\
\hline & mid-tibial diameter [mm] & $2.813 \pm 0.150$ & $2.833 \pm 0.151$ & $2.627 \pm 0.116^{*}$ \\
\hline
\end{tabular}

Results are presented as mean \pm standard deviation (SD); $\mathrm{p}<0.05$ compared to the control group; ANOVA - analysis of variance; LSD - least significant difference.

Table 2. The effect of long-term administration of efavirenz and tenofovir on bone mineral density (one-way ANOVA with post hoc LSD test)

\begin{tabular}{|c|c|c|c|c|}
\hline & Parameter & $\begin{array}{l}\text { Group C } \\
(n=12)\end{array}$ & $\begin{array}{c}\text { Group EF } \\
(n=12)\end{array}$ & $\begin{array}{l}\text { Group T } \\
(n=12)\end{array}$ \\
\hline \multirow{3}{*}{ Week 12} & total body BMD $\left[\mathrm{g} / \mathrm{cm}^{2}\right]$ & $0.2122 \pm 0.0040$ & $0.2101 \pm 0.0046$ & $0.2067 \pm 0.0122$ \\
\hline & femoral BMD [g/cm²] & $0.3155 \pm 0.0320$ & $0.3077 \pm 0.0191$ & $0.3088 \pm 0.0281$ \\
\hline & tibial BMD $\left[\mathrm{g} / \mathrm{cm}^{2}\right]$ & $0.2425 \pm 0.0174$ & $0.2316 \pm 0.0264$ & $0.2332 \pm 0.0290$ \\
\hline \multirow{4}{*}{ Week 24} & total body BMD [g/cm²] & $0.2272 \pm 0.0096$ & $0.22 \pm 0.0078$ & $0.2146 \pm 0.0066^{*}$ \\
\hline & femoral BMD $\left[\mathrm{g} / \mathrm{cm}^{2}\right]$ & $0.3836 \pm 0.0167$ & $0.3785 \pm 0.0194$ & $0.371 \pm 0.022$ \\
\hline & tibial BMD $\left[\mathrm{g} / \mathrm{cm}^{2}\right]$ & $0.2606 \pm 0.0116$ & $0.2518 \pm 0.0112$ & $0.2516 \pm 0.0152$ \\
\hline & L1-L4 spine BMD $\left[\mathrm{g} / \mathrm{cm}^{2}\right]$ & $0.3565 \pm 0.0283$ & $0.3548 \pm 0.0235$ & $0.3382 \pm 0.0280$ \\
\hline
\end{tabular}

Results are presented as mean \pm standard deviation (SD); ${ }^{*} \mathrm{p}<0.05$ compared to the control group; BMD - bone mineral density; ANOVA - analysis of variance; LSD - least significant difference. 

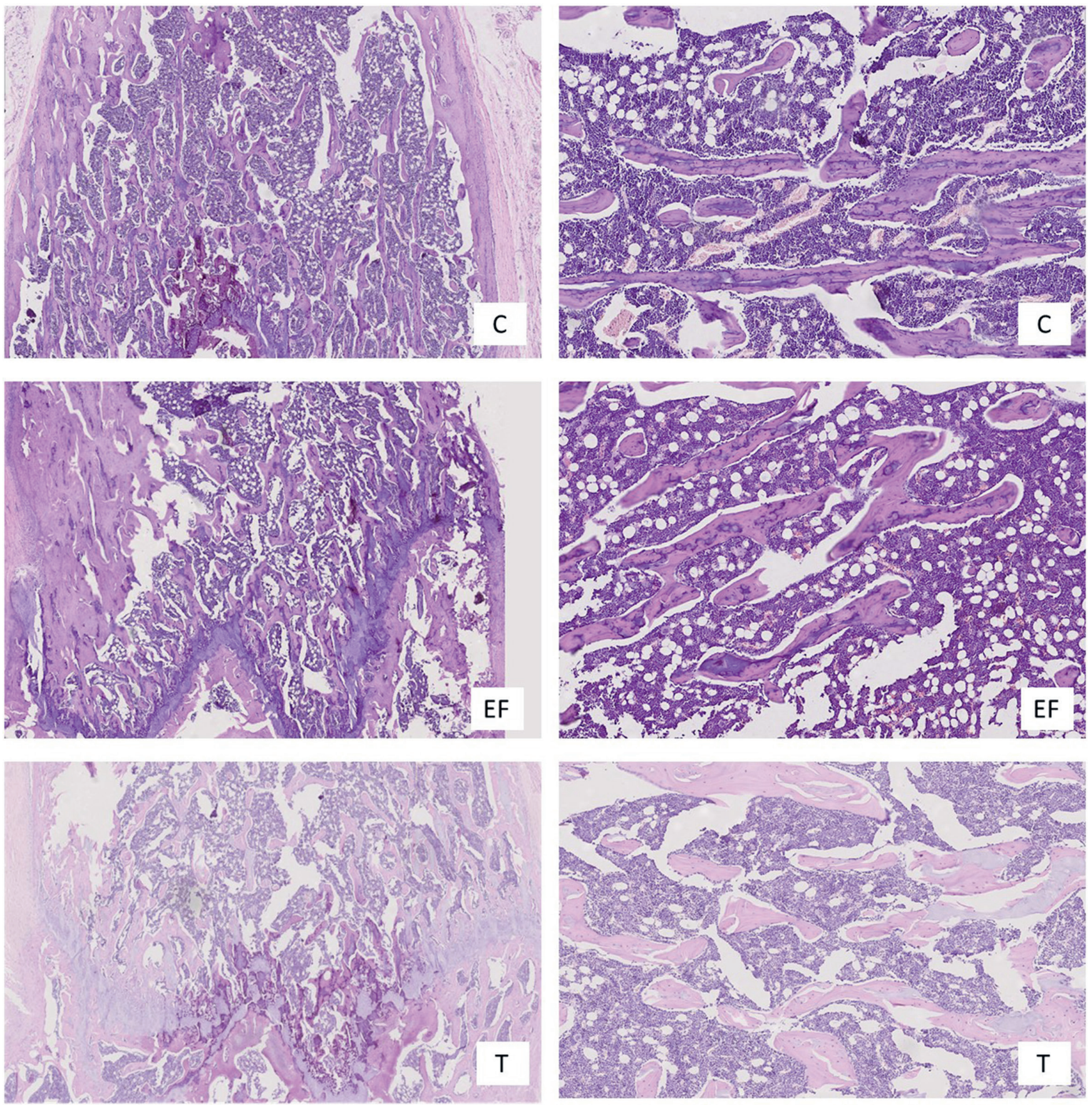

Fig. 3. Sample images of the histopathological examination (H\&E staining) of the distal metaphysis of the femur in groups $C$, EF and $T$ (magnification $\times 15$ and $\times 50$ )

\section{Bone biomechanical properties}

The results are presented in Table 5. The four-point bending test demonstrated a lower value of Young's modulus in groups EF and $\mathrm{T}$ than in group $\mathrm{C}(\mathrm{p}=0.037$, $\mathrm{p}=0.006$, respectively).

\section{Discussion}

The aim of this study was to assess the effect of 2 antiretroviral medications - EF and $\mathrm{T}$ - on growing skeletons.
Abnormal increases in bone mass during the critical period of development of the skeleton may lead to disorders in bone growth and/or lower adult peak bone mass. Lower peak bone mass increases the risk of osteoporosis and fractures occurring later in life. ${ }^{29}$

Insulin-like growth factor 1 participates in the regulation of the growing process, as well in increasing and maintaining bone mass. It constitutes the main mediator in the action of the growth hormone on target cells, mainly on chondrocytes, osteoblasts and endocrine cells. ${ }^{30}$ This study failed to demonstrate the effect of the substances studied (EF and T) on the level of IGF-1, which suggests 
A
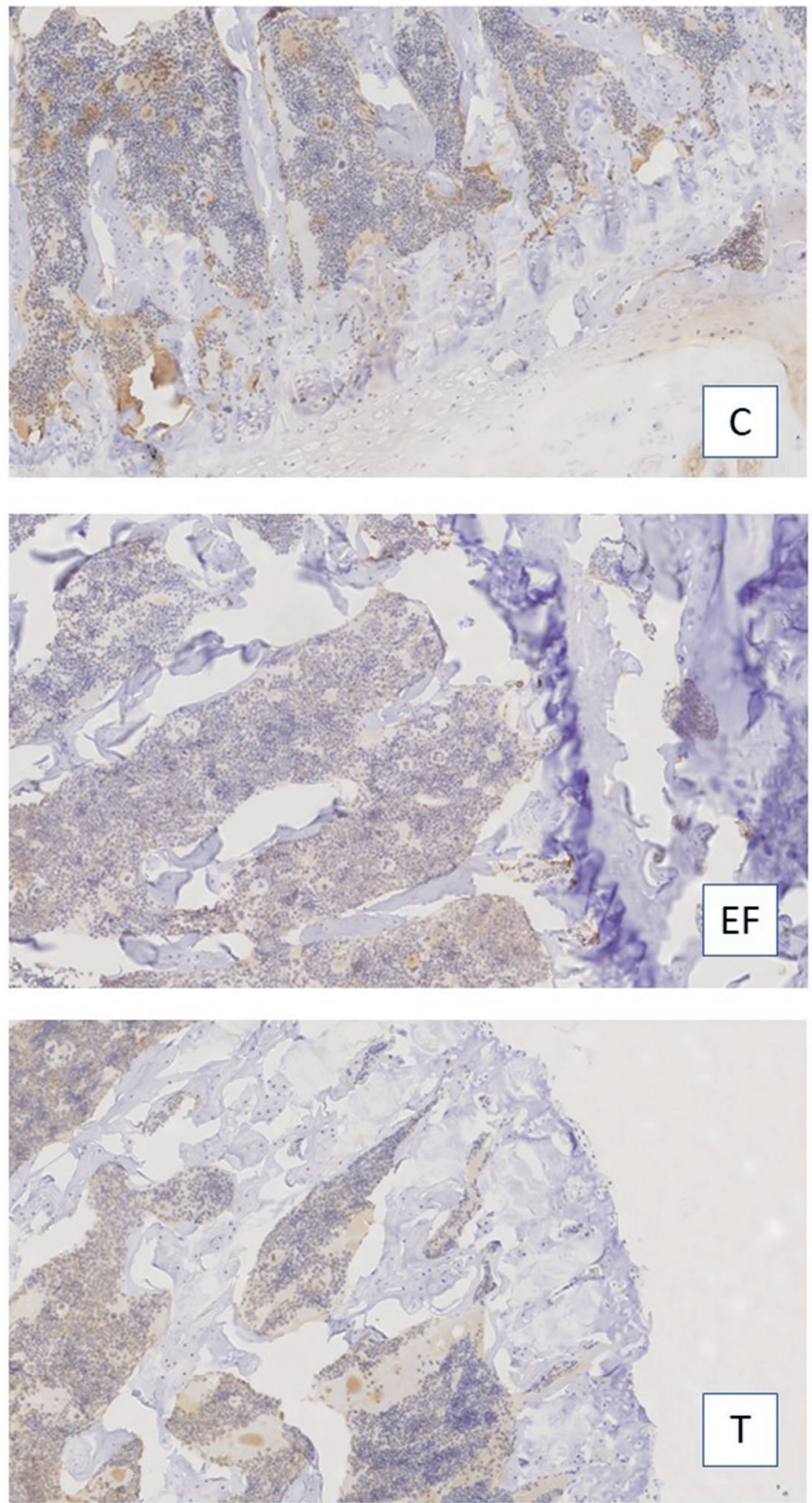

B
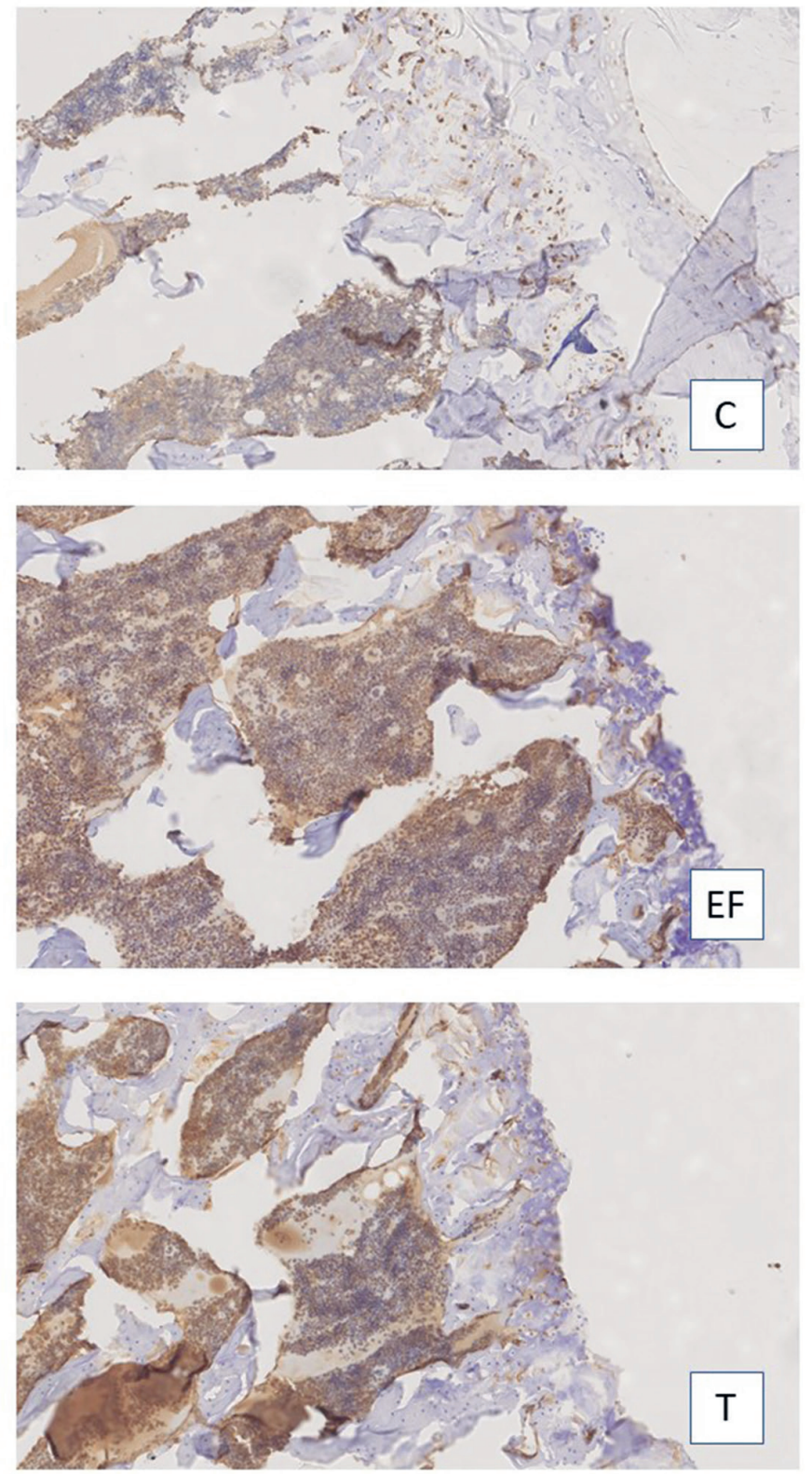

Fig. 4. Sample images of the immunohistochemical examination ( - - abTRACP staining; $B-a b A P$ staining) of the $L 2$ in groups $C$, EF and $\mathrm{T}$ (magnification $\times 10)$

that the effect of those substances on bone does not depend on IGF-1.

In this study, we observed lower femoral and tibial indices in group EF, which indicates lower relative dimensions of the examined bones in this group. However, we did not observe reduced BMD of the bones analyzed, which is consistent with the results from Arpadi et al. ${ }^{18}$ Our histopathological examination of the L2 demonstrated a reduction in BS/BV. A 36\% reduction in the number of trabeculae accompanied by a higher trabecular thickness (Tb.Th) was observed at the same time. The absence of significant changes in the histopathological examination of femurs and tibias in the group EF could be attributed to a more rapid remodeling of osseous tissue in the spine as compared to long bones. ${ }^{31}$
An increased serum level of TRAP and increased abTRAP expression in our immunohistochemical examination of L2 were observed in the group EF, indicating an increased number and increased activity of osteoclasts ${ }^{32}$ in this group. The influence of the high SD of abTRAP (in both intensity and extension) on the obtained results cannot be excluded. However, as the serum concentration of TRAP corresponds to the immunohistochemical results, it does not affect our overall conclusion on the influence of $\mathrm{T}$ on bone turnover. At the same time, EF decreased levels of osteocalcin, a marker of bone formation. This suggests that the changes observed in bone microarchitecture in the group EF could result from increased activity of resorption and decreased bone formation. In the animals receiving EF, phosphorus 
Table 3. The effects of long-term administration of efavirenz and tenofovir on histopathological and immunohistochemical parameters of bone (one-way ANOVA with post hoc LSD test)

\begin{tabular}{|c|c|c|c|c|}
\hline & neter & $\begin{array}{l}\text { Group C } \\
(n=12)\end{array}$ & $\begin{array}{l}\text { Group EF } \\
(\mathrm{n}=12)\end{array}$ & $\begin{array}{l}\text { Group T } \\
(n=12)\end{array}$ \\
\hline \multirow{10}{*}{ L2 vertebra } & abTRAP - intensity & $0.625 \pm 1.188$ & $1.778 \pm 1.394^{*}$ & $0.1818 \pm 0.603$ \\
\hline & abTRAP - extension & $0.375 \pm 0.744$ & $1.111 \pm 0.928 *$ & $0.0909 \pm 0.3015$ \\
\hline & abAP - intensity & $3.0 \pm 0.0$ & $3.0 \pm 0.0$ & $3.0 \pm 0.0$ \\
\hline & abAP - extension & $1.875 \pm 0.991$ & $2.0 \pm 0.8165$ & $2.545 \pm 0.5222$ \\
\hline & $\mathrm{BV} / \mathrm{TV}[\%]$ & $0.1391 \pm 0.0088$ & $0.2157 \pm 0.2386$ & $0.1345 \pm 0.0058$ \\
\hline & $\mathrm{BS} / \mathrm{BV}\left[\mathrm{mm}^{2} / \mathrm{mm}^{3}\right]$ & $68.57 \pm 41.93$ & $36.727 \pm 24.659^{*}$ & $52.14 \pm 4.913$ \\
\hline & $\mathrm{BS} / \mathrm{TV}\left[\mathrm{mm}^{2} / \mathrm{mm}^{3}\right]$ & $9.616 \pm 5.859$ & $6.145 \pm 3.785$ & $7.006 \pm 0.656$ \\
\hline & Tb.Th [mm] & $0.0455 \pm 0.0365$ & $0.0752 \pm 0.0391^{*}$ & $0.0387 \pm 0.0034$ \\
\hline & Tb.N [1/mm] & $4.808 \pm 2.929$ & $3.072 \pm 1.892$ & $3.503 \pm 0.3279$ \\
\hline & Tb.Sp [mm] & $0.2832 \pm 0.2617$ & $0.3777 \pm 0.2649$ & $0.2518 \pm 0.02944$ \\
\hline \multirow{6}{*}{ Femur } & $\mathrm{BV} / \mathrm{TV}[\%]$ & $0.2512 \pm 0.0473$ & $0.2621 \pm 0.1519$ & $0.1899 \pm 0.0763$ \\
\hline & $\mathrm{BS} / \mathrm{BV}\left[\mathrm{mm}^{2} / \mathrm{mm}^{3}\right]$ & $46.724 \pm 17.336$ & $40.395 \pm 12.937$ & $44.11 \pm 7.858$ \\
\hline & $\mathrm{BS} / \mathrm{TV}\left[\mathrm{mm}^{2} / \mathrm{mm}^{3}\right]$ & $11.811 \pm 5.083$ & $9.035 \pm 1.551$ & $7.962 \pm 1.906$ \\
\hline & Tb.Th [mm] & $0.0471 \pm 0.0134$ & $0.0582 \pm 0.0333$ & $0.0468 \pm 0.0092$ \\
\hline & Tb.N [1/mm] & $5.906 \pm 2.542$ & $4.517 \pm 0.7757$ & $3.981 \pm 0.9531 *$ \\
\hline & Tb.Sp [mm] & $0.1471 \pm 0.05531$ & $0.1687 \pm 0.05228$ & $0.2174 \pm 0.06817^{*}$ \\
\hline \multirow{6}{*}{ Tibia } & $\mathrm{BV} / \mathrm{TV}[\%]$ & $0.2398 \pm 0.0491$ & $0.2007 \pm 0.0332$ & $0.2044 \pm 0.04270$ \\
\hline & $\mathrm{BS} / \mathrm{BV}\left[\mathrm{mm}^{2} / \mathrm{mm}^{3}\right]$ & $44.661 \pm 17.357$ & $44.223 \pm 6.283$ & $43.96 \pm 6.231$ \\
\hline & $\mathrm{BS} / \mathrm{TV}\left[\mathrm{mm}^{2} / \mathrm{mm}^{3}\right]$ & $10.339 \pm 3.375$ & $8.778 \pm 1.252$ & $8.812 \pm 1.165$ \\
\hline & Tb.Th [mm] & $0.0496 \pm 0.0149$ & $0.0460 \pm 0.0059$ & $0.0463 \pm 0.0065$ \\
\hline & Tb.N $[1 / \mathrm{mm}]$ & $5.17 \pm 1.688$ & $4.389 \pm 0.6261$ & $4.406 \pm 0.5827$ \\
\hline & Tb.Sp [mm] & $0.1580 \pm 0.04195$ & $0.1856 \pm 0.02811$ & $0.1842 \pm 0.03210$ \\
\hline
\end{tabular}

Results are presented as mean \pm standard deviation (SD); ${ }^{*} \mathrm{p}<0.05$ compared to the control group; L2 - second lumbar vertebra; abTRAP - anti-tartrate resistant acid phosphatase antibody; abAP - recombinant anti-alkaline phosphatase antibody; BV - bone volume; TV - tissue volume; BV/TV - tissue volume ratio; BS - bone surface; Tb.Th - trabecular thickness; Tb.N - trabecular number; Tb.Sp - trabecular separation; ANOVA - analysis of variance; LSD - least significant difference.

Table 4. The effects of long-term administration of efavirenz and tenofovir on serum parameters (one-way ANOVA with post hoc LSD test)

\begin{tabular}{|c|c|c|c|}
\hline Parameter & $\begin{array}{l}\text { Group C } \\
(n=12)\end{array}$ & $\begin{array}{l}\text { Group EF } \\
(\mathrm{n}=12)\end{array}$ & $\begin{array}{l}\text { Group T } \\
(n=12)\end{array}$ \\
\hline IGF-1 [ng/mL] & $3.0183 \pm 0.8979$ & $4.5537 \pm 3.5369$ & $2.5656 \pm 1.2765$ \\
\hline Osteocalcin [pg/mL] & $193.865 \pm 46.260$ & $138.022 \pm 45.965^{*}$ & $223.857 \pm 45.200$ \\
\hline $\mathrm{CTX}[\mathrm{ng} / \mathrm{mL}]$ & $17.759 \pm 4.337$ & $19.299 \pm 9.259$ & $17.883 \pm 4.662$ \\
\hline $\operatorname{TRAP}[U / L]$ & $0.776 \pm 0.265$ & $1.088 \pm 0.264^{*}$ & $0.843 \pm 0.165$ \\
\hline 1,25-hydoxy-vitamin D3 [nmol/L] & $1.52 \pm 0.16$ & $1.40 \pm 0.32$ & $1.46 \pm 0.22$ \\
\hline Total calcium [mg/dL] & $6.427 \pm 1.769$ & $8.192 \pm 1.358^{*}$ & $6.250 \pm 1.731$ \\
\hline Inorganic phosphorus [mg/dL] & $3.618 \pm 1.018$ & $5.683 \pm 1.210^{*}$ & $3.608 \pm 1.453$ \\
\hline Alkaline phosphatase [U/L] & $56.363 \pm 19.185$ & $64.333 \pm 16.041$ & $60.300 \pm 24.891$ \\
\hline Creatinine [mg/dL] & $0.303 \pm 0.051$ & $0.324 \pm 0.075$ & $0.358 \pm 0.105$ \\
\hline
\end{tabular}

Results are presented as mean \pm standard deviation (SD); ${ }^{*} \mathrm{p}<0.05$ compared to the control group CTX - beta C-terminated telopeptide of type I collagen; TRAP - osteoclast-derived tartrate-resistant acid phosphatase form 5b; IGF-1 - insulin-like growth factor 1; ANOVA - analysis of variance; LSD - least significant difference.

and calcium levels were higher compared to group C. The observed changes may be a consequence of increased osteoclast activity and increased bone resorption. Several studies have demonstrated decreases in vitamin D levels in patients treated with NNRTIs. ${ }^{33-35}$ However, we did not detect decreases in vitamin D levels in our study. It is hypothesized that decreases in vitamin $D$ levels in patients treated with efavirenz may stem from induction of the cytochrome P450 (CYP24A), which is responsible for the breakdown of active vitamin D in humans, ${ }^{36}$ but not in rats. ${ }^{37}$ 
Table 5. The effects of long-term administration of efavirenz and tenofovir on biomechanical parameters of the right femur (one-way ANOVA with post hoc LSD test)

\begin{tabular}{|l|c|c|c|}
\multicolumn{1}{|c|}{ Parameter } & Group C & Group EF & Group T \\
\hline Young's modulus [GPa] & $16.216 \pm 3.014$ & $12.989 \pm 2.391^{*}$ & $13.872 \pm 1.927^{*}$ \\
\hline Flexural strength [MPa] & $98.881 \pm 30.608$ & $85.633 \pm 25.961$ & $105.285 \pm 19.296$ \\
\hline Rigidity [Nm] & $0.2823 \pm 0.0441$ & $0.2406 \pm 0.0665$ & $0.2438 \pm 0.0664$ \\
\hline
\end{tabular}

Results are presented as mean \pm standard deviation (SD); ${ }^{*} p<0.05$ compared to the control group; ANOVA - analysis of variance; LSD - least significant difference.

In group EF, besides the reductions in femoral indices and disturbances of bone microarchitecture demonstrated in the histological examination, a 20\% reduction of Young's modulus was found in the four-point bending test, which is a sign of deterioration of bone biomechanical properties that may lead to increased risk of fractures.

Reduced femoral indices and mid-femoral diameters were also found in group $\mathrm{T}$, as in group EF. However, no effect of $\mathrm{T}$ on tibial indices was observed. Moreover, a reduction in TB BMD was found in group T. These results are consistent with the observations of Gafni et al. and Purdy et al., who found reduced BMD in T-treated patients. ${ }^{20,38}$ In their studies of growing rhesus monkeys, Castillo et al. demonstrated a mineralization defect in newly-formed cortical bones in monkeys receiving $\mathrm{T}$, leading to the formation of completely non-mineralized osteons. ${ }^{39}$ This is consistent with the observation of a lower percentage of mineralizing surfaces in inflammation-free rats receiving $\mathrm{T}$ in a study by Conradie et al. ${ }^{40}$ The results of our study also suggest bone mineralization disorders during treatment with T. Clinical studies also report greater decreases in BMD associated with $\mathrm{T}$ therapy. ${ }^{41-45}$ Besides the reduction in TB BMD, expression of recombinant antialkaline phosphatase (anti-AP) was extended by $36 \%$ in L2 in group $\mathrm{T}$. Moreover, reductions in femoral BS/TV and in the number of trabeculae (Tb.N) with simultaneous increases in $\mathrm{Tb}$.Sp were observed in group $\mathrm{T}$. Changes like this in bone histomorphometry were not reported by Ramalho et al. ${ }^{44}$ Some authors have suggested that T-associated decreases in BMD may be a consequence of renal failure and subsequent decreases in vitamin D activation, but in our study we detected neither an increase in creatinine level nor a decrease in vitamin D level. Our results are consistent with the report by Bagger et al. suggesting that T-associated decreases in BMD cannot be explained by decreased renal function. ${ }^{46}$

The pathogenesis of bone lesions occurring during treatment with $\mathrm{T}$ is poorly understood and needs to be further elucidated. A direct effect of the drug on osteoclasts and/or osteoblasts is considered, as well as an indirect effect resulting from injury to the proximal renal tubules, hypophosphatemia and an increased release of parathyroid hormones. ${ }^{47}$ Serum phosphate levels may not reflect TB phosphate depletion, nor even re-absorption of phosphates into the renal tubules. ${ }^{48}$ Despite the significant reductions in BMD and changes in bone microarchitecture observed in group $\mathrm{T}$, our study did not demonstrate the effect of $\mathrm{T}$ on serum inorganic phosphorus levels, nor on any other recognized markers of bone turnover. Therefore, the pathomechanism of the observed phenomenon needs to be studied further.

Decreased biomechanical properties of bones expressed by the $14 \%$ reduction in Young's modulus were observed in group $\mathrm{T}$ in the four-point bending test. Conradie et al. reported that biomechanical properties of femurs from non-HIV infected rats were not statistically different between group $\mathrm{T}$ and group $\mathrm{C} .{ }^{40}$ This could be a consequence of the fact that in their study a threepoint bending test was performed. The four-point bending test may be more sensitive, but further biomechanical experiments are required to verify this hypothesis. The four-point bending method differs from three-point bending due to the way the measurements are carried out. The three-point bending test, compared to four-point bending, underestimates the modulus of elasticity due to the fact that the shear effect and the indentation effect of the loading head and the supports are neglected. A four-point test tends to be the best choice, especially if the examined material is (like bones) not homogeneous. The stress of a three-point test is concentrated under the center of the loading point, whereas the stress concentration of a four-point test is over a larger region. We have already introduced this method in several other studies, ${ }^{49,50}$ and it gives very good results, especially since it does not cause permanent bone deformation in the places of where force is directly applied. The bending energy is therefore directly related only to the fracture site. Thus, the results obtained are characterized by higher values of the mechanical parameters and a smaller range of results in comparison to the three-point test.

\section{Conclusions}

In a controlled environment where HIV-associated factors were absent, the long-term monotherapy with EF and tenofovir disoproxil had an adverse effect on the bones of growing male rats. The results of this study indicate that EF affects bone microarchitecture and leads to impaired bone biomechanical properties - an effect that seems to be associated with increased activity of osteoclasts and decreased new bone formation. 


\section{ORCID iDs}

Agnieszka Matuszewska (D) https://orcid.org/0000-0003-1082-0793 Beata Nowak (D) https://orcid.org/0000-0003-0014-6344 Anna Nikodem (D) https://orcid.org/0000-0002-1418-247X Diana Jędrzejuk (D) https://orcid.org/0000-0001-7053-142X Danuta Szkudlarek (D) https://orcid.org/0000-0001-5736-310X Krzysztof Zduniak (D) https://orcid.org/0000-0003-4652-4478 Jarosław Filipiak (D) https://orcid.org/0000-0002-9380-0642 Marta Sznadruk-Bender (D) https://orcid.org/0000-0002-4571-9452 Tomasz Tomkalski (D) https://orcid.org/0000-0003-2850-7316 Ireneusz Ceremuga (iD https://orcid.org/0000-0001-5744-2860 Marek Bolanowski (D) https://orcid.org/0000-0002-2360-6596 Adam Szeląg (D) https://orcid.org/0000-0001-8104-5267

\section{References}

1. UNAIDS. 2017 Global HIV Statistics. Fact sheet. Geneva, Switzerland: The Joint United Nations Programme on HIV and AIDS; 2017.

2. Shiau S, Yin MT, Strehlau R, et al. Decreased bone turnover in HIVinfected children on antiretroviral therapy. Arch Osteoporos. 2018; 13(1):40. doi:10.1007/s11657-018-0452-6

3. Zuccotti G, Viganò A, Gabiano C, et al. Antiretroviral therapy and bone mineral measurements in HIV-infected youths. Bone. 2010; 46(6):1633-1638. doi:10.1016/j.bone.2010.02.029

4. Margossian R, Williams PL, Yu W, et al; Pediatric HIV/AIDS Cohort Study (PHACS). Markers of bone mineral metabolism and cardiac structure and function in perinatally HIV-infected and HIV-exposed but uninfected children and adolescents. J Acquir Immune Defic Syndr. 2019;81(2):238-246. doi:10.1097/QAI.0000000000002007

5. Jacobson DL, Stephensen CB, Miller TL, et al; Pediatric HIV/AIDS Cohort Study. Associations of low vitamin $D$ and elevated parathyroid hormone concentrations with bone mineral density in perinatally HIV-infected children. J Acquir Immune Defic Syndr. 2017;76(1): 33-42. doi:10.1097/QAl.0000000000001467

6. Manavalan JS, Arpadi S, Tharmarajah S, et al. Abnormal bone acquisition with early-life HIV infection: Role of immune activation and senescent osteogenic precursors. J Bone Miner Res. 2016;31(11):1988-1996. doi:10.1002/jbmr.2883

7. Eckard AR, Mora S. Bone health in HIV-infected children and adolescents. Curr Opin HIV AIDS. 2016;11(3):294-300. doi:10.1097/COH. 0000000000000270

8. Vikulina T, Fan X, Yamaguchi M, et al. Alterations in the immunoskeletal interface drive bone destruction in HIV-1 transgenic rats. Proc Natl Acad Sci U S A. 2010;107(31):13848-13853. doi:10.1073/pnas. 1003020107

9. Ofotokun I. Deciphering how HIV-1 weakens and cracks the bone. Proc Natl Acad Sci U S A. 2018;115(11):2551-2553. doi:10.1073/pnas. 1801555115

10. Biver E, Calmy A, Rizzoli R. Bone health in HIV and hepatitis B or C infections. Ther Adv Musculoskelet Dis. 2016;9(1):22-34. doi:10.1177/ $1759720 \times 16671927$

11. Brown TT, Qaqish RB. Antiretroviral therapy and the prevalence of osteopenia and osteoporosis: A meta-analytic review. AIDS. 2006; 20(17):2165-2174. doi:10.1097/QAD.0b013e32801022eb

12. Madeddu G, Spanu A, Solinas P, et al. Different impact of NNRTI and PI-including HAART on bone mineral density loss in HIV-infected patients. Eur Rev Med Pharmacol Sci. 2015;19(23):4576-4589.

13. Tebas $P$, Kumar $P$, Hicks $C$, et al. Greater change in bone turnover markers for efavirenz/emtricitabine/tenofovir disoproxil fumarate versus dolutegravir + abacavir/lamivudine in antiretroviral therapy-naive adults over 144 weeks. AIDS. 2015;29(18):2459-2464. doi:10.1097/ QAD.0000000000000863

14. Ofotokun I, Titanji K, Vunnava A, et al. Antiretroviral therapy induces a rapid increase in bone resorption that is positively associated with the magnitude of immune reconstitution in HIV infection. AIDS. 2016;30(3):405-414. doi:10.1097/QAD.0000000000000918

15. Yin MT, Chan ES, Brown TT, et al. Vitamin D does not modulate immune-mediated bone loss during ART initiation. Antivir Ther. 2019; 24(5):355-362. doi:10.3851/IMP3316

16. McComsey GA, Kitch D, Daar ES, et al. Bone mineral density and fractures in antiretroviral-naive persons randomized to receive abacavir-lamivudine or tenofovir disoproxil fumarate-emtricitabine along with efavirenz or atazanavir-ritonavir: AIDS Clinical Trials Group
A5224s, a substudy of ACTG. J Infect Dis. 2011;203(12):1791-1801. doi:10.1093/infdis/jir188

17. Aurpibul L, Puthanakit T. Review of tenofovir use in HIV-infected children. Pediatr Infect Dis J. 2015;34(4):383-391. doi:10.1097/INF. 0000000000000571

18. Arpadi SM, Shiau S, Strehlau R, et al. Efavirenz is associated with higher bone mass in South African children with HIV. AIDS. 2016;30(16): 2459-2467. doi:10.1097/QAD.0000000000001204

19. Dave JA, Cohen K, Micklesfield LK, Maartens G, Levitt NS. Antiretroviral therapy, especially efavirenz, is associated with low bone mineral density in HIV-infected South Africans. PLoS One. 2015;10(12): e0144286. doi:10.1371/journal.pone.0144286

20. Gafni RI, Hazra R, Reynolds JC, et al. Tenofovir disoproxil fumarate and an optimized background regimen of antiretroviral agents as salvage therapy: Impact on bone mineral density in HIV-infected children. Pediatrics. 2006;118(3):e711-e718. doi:10.1542/peds. 2005-2525

21. Viganò A, Zuccotti GV, Puzzovio M, et al. Tenofovir disoproxil fumarate and bone mineral density: $\mathrm{A}$ 60-month longitudinal study in a cohort of HIV-infected youths. Antivir Ther. 2010;15(7):1053-1058. doi:10.3851/IMP1650

22. Unsal AB, Mattingly AS, Jones SE, et al. Effect of antiretroviral therapy on bone and renal health in young adults infected with HIV in early life. J Clin Endocrinol Metab. 2017;102(8):2896-2904. doi:10.1210/ jc.2017-00197

23. Hernandez CJ, Beaupré GS, Carter DR. A theoretical analysis of the changes in basic multicellular unit activity at menopause. Bone. 2003; 32(4):357-363. doi:10.1016/s8756-3282(03)00037-1

24. Compston J. HIV infection and bone disease. J Intern Med. 2016; 280(4):350-358. doi:10.1111/joim.12520

25. Goffinet C, Allespach I, Keppler OT. HIV-susceptible transgenic rats allow rapid preclinical testing of antiviral compounds targeting virus entry or reverse transcription. Proc Natl Acad Sci. 2007;104(3):1015-1020. doi:10.1073/pnas.0607414104

26. Watkins ME, Wring S, Randolph $\mathrm{R}$, et al. Development of a novel formulation that improves preclinical bioavailability of tenofovir disoproxil fumarate. J Pharm Sci. 2017;106(3):906-919. doi:10.1016/j. xphs.2016.12.003

27. Dempster DW, Compston JE, Drezner MK, et al. Standardized nomenclature, symbols, and units for bone histomorphometry: A 2012 update of the report of the ASBMR Histomorphometry Nomenclature Committee. J Bone Miner Res. 2013;28(1):2-17. doi:10.1002/jbmr.1805

28. Torlakovic EE, Francis G, Garratt J, et al; International Ad Hoc Expert Panel. Standardization of negative controls in diagnostic immunohistochemistry: Recommendations from the international ad hoc expert panel. Appl/mmunohistochem MolMorphol. 2014;22(4):241-252. doi:10.1097/PAl.0000000000000069

29. Tandon N, Fall CHD, Osmond C, et al. Growth from birth to adulthood and peak bone mass and density data from the New Delhi Birth Cohort. Osteoporos Int. 2012;23(10):2447-2459. doi:10.1007/s00198011-1857-x

30. Filus A, Zdrojewicz Z. Insulin-like growth factor-1 (IGF-1) - structure and the role in the human body [in Polish]. Pediatr Endocrinol Diabetes Metab. 2014;20(4):161-169. doi:10.18544/PEDM-20.04.0016

31. Datta HK, Ng WF, Walker JA, Tuck SP, Varanasi SS. The cell biology of bone metabolism. J Clin Pathol. 2008;61(5):577-587. doi:10.1136/ JCP.2007.048868

32. Hlaing TT, Compston JE. Biochemical markers of bone turnover: Uses and limitations. Ann Clin Biochem. 2014;51(Pt 2):189-202. doi:10.1177/ 0004563213515190

33. Vescini F, Cozzi-Lepri A, Borderi M, et al; Icona Foundation Study Group. Prevalence of hypovitaminosis D and factors associated with vitamin D deficiency and morbidity among HIV-infected patients enrolled in a large Italian cohort. J Acquir Immune Defic Syndr. 2011; 58(2):163-172. doi:10.1097/QAl.0b013e31822e57e9

34. Conesa-Botella A, Florence E, Lynen L, Colebunders R, Menten J, Moreno-Reyes R. Decrease of vitamin D concentration in patients with HIV infection on a non-nucleoside reverse transcriptase inhibitor-containing regimen. AIDS Res Ther. 2010;7:40. doi:10.1186/17426405-7-40

35. Welz T, Childs K, Ibrahim F, et al. Efavirenz is associated with severe vitamin D deficiency and increased alkaline phosphatase. AIDS. 2010; 24(12):1923-1928. doi:10.1097/QAD.0b013e32833c3281 
36. Guryev O, Carvalho RA, Usanov S, Gilep A, Estabrook RW. A pathway for the metabolism of vitamin D3: Unique hydroxylated metabolites formed during catalysis with cytochrome P450scc (CYP11A1). Proc Natl Acad Sci U S A. 2003;100(25):14754-14759. doi:10.1073/pnas. 2336107100

37. Horst RL, Omdahl JA, Reddy S. Rat cytochrome P450C24 (CYP24) does not metabolize 1,25-dihydroxyvitamin D 2 to calcitroic acid. J Cell Biochem. 2003;88(2):282-285. doi:10.1002/jcb.10359

38. Purdy JB, Gafni RI, Reynolds JC, Zeichner S, Hazra R. Decreased bone mineral density with off-label use of tenofovir in children and adolescents infected with human immunodeficiency virus. J Pediatr. 2008; 152(4):582-584. doi:10.1016/j.jpeds.2007.12.020

39. Castillo AB, Tarantal AF, Watnik MR, Bruce Martin R. Tenofovir treatment at $30 \mathrm{mg} / \mathrm{kg} /$ day can inhibit cortical bone mineralization in growing rhesus monkeys (Macaca mulatta). J Orthop Res. 2002;20(6): 1185-1189. doi:10.1016/S0736-0266(02)00074-8

40. Conradie MM, van de Vyver M, Andrag E, Conradie M, Ferris WF. A direct comparison of the effects of the antiretroviral drugs stavudine, tenofovir and the combination lopinavir/ritonavir on bone metabolism in a rat model. Calcif Tissue Int. 2017;101(4):422-432. doi:10.1007/s00223-017-0290-3

41. Gallant JE, Staszewski S, Pozniak AL, et al. Efficacy and safety of tenofovir DF vs stavudine in combination therapy in antiretroviralnaive patients: A 3-year randomized trial. JAMA. 2004;292(2):191-201. doi:10.1001/jama.292.2.191

42. Stellbrink H, Orkin C, Arribas JR, et al; ASSERT Study Group. Comparison of changes in bone density and turnover with abacavir-lamivudine versus tenofovir-emtricitabine in HIV-infected adults: 48-week results from the ASSERT study. Clin Infect Dis. 2010;51(8):963-972. doi:10.1086/656417

43. Hamzah L, Tiraboschi JM, Iveson H, et al. Effects on vitamin D, bone and the kidney of switching from fixed-dose tenofovir disoproxil fumarate/emtricitabine/efavirenz to darunavir/ritonavir monotherapy: A randomized, controlled trial (MIDAS). Antivir Ther. 2016;21(4): 287-296. doi:10.3851/IMP3000
44. Ramalho J, Martins CSW, Galvão J, et al. Treatment of human immunodeficiency virus infection with tenofovir disoproxil fumarate-containing antiretrovirals maintains low bone formation rate, but increases osteoid volume on bone histomorphometry. J Bone Miner Res. 2019;34(9):1574-1584. doi:10.1002/jbmr.3751

45. Venter WDF, Kambugu A, Chersich MF, et al. Efficacy and safety of tenofovir disoproxil fumarate versus low-dose stavudine over 96 weeks: A multicountry randomized, noninferiority trial. J Acquir Immune Defic Syndr. 2019;80(2):224-233. doi:10.1097/QAI.000000000 0001908

46. Bagger $Y Z$, Rasmussen $H B$, Alexandersen $P$, Werge $T$, Christiansen $C$, Tankó LB; PERF study group. Links between cardiovascular disease and osteoporosis in postmenopausal women: Serum lipids or atherosclerosis per se? Osteoporos Int. 2007;18(4):505-512. doi:10.1007/ s00198-006-0255-2

47. Grigsby IF, Pham L, Mansky LM, Gopalakrishnan R, Mansky KC. Tenofovir-associated bone density loss. Ther Clin Risk Manag. 2010;6(1): 41-47. doi:10.2147/TCRM.S8836

48. Casado JL. Renal and bone toxicity with the use of tenofovir: Understanding at the end. AIDS Rev. 2016;18(2):59-68.

49. Nowak B, Matuszewska A, Filipiak J, et al. The influence of bexarotene, a selective agonist of the retinoid receptor $X(R X R)$, and tazarotene, a selective agonist of the retinoid acid receptor (RAR), on bone metabolism in rats. Adv Med Sci. 2016;61(1):85-89. doi:10.1016/j. advms.2015.09.001

50. Markiewicz-Górka I, Kuropka P, Januszewska L, et al. Influence of physical training on markers of bone turnover, mechanical properties, morphological alterations, density and mineral contents in the femur of rats exposed to cadmium and/or alcohol. Toxicol Ind Health. 2019;35(4):277-293. doi:10.1177/0748233719831534 